\title{
Non-organic hearing loss: new and confirmed findings
}

\author{
Alexandra Holenweg $\cdot$ Martin Kompis
}

Received: 19 November 2009/Accepted: 9 February 2010/Published online: 4 March 2010

(C) Springer-Verlag 2010

\begin{abstract}
Although non-organic hearing losses are relatively rare, it is important to identify suspicious findings early to be able to administer specific tests, such as objective measurements and specific counseling. In this retrospective study, we searched for findings that were specific ti or typical for non-organic hearing losses. Patient records from a 6 year period (2003-2008) from the University ENT Department of Bern, Switzerland, were reviewed. In this period, 40 subjects were diagnosed with a non-organic hearing loss (22 children, ages 7-16, mean 10.6 years; 18 adults, ages $19-57$, mean 39.7 years; 25 females and 15 males). Pure tone audiograms in children and adults showed predominantly sensorineural and frequency-independent hearing losses, mostly in the range of 40-60 dB. In all cases, objective measurements (otoacoustic emissions and/or auditory-evoked potentials) indicated normal or substantially better hearing thresholds than those found in pure tone audiometry. In nine subjects $(22.5 \% ; 2$ children, 7 adults), hearing aids had been fitted before the first presentation at our center. Six children (27\%) had a history of middle ear problems with a transient hearing loss and $11(50 \%)$ knew a person with a hearing loss. Two new and hitherto unreported findings emerged from the analysis: it was observed that a small air-bone gap of 5-20 dB was typical for non-organic hearing losses and that speech audiometry might show considerably poorer results than expected from pure tone audiometry.
\end{abstract}

Keywords Pseudohypacusis - Non-organic hearing loss . Aggravation · Malingering · Psychogenic hearing loss

A. Holenweg · M. Kompis $(\square)$

Department of ENT, Head and Neck Surgery, Inselspital,

University of Bern, 3010 Bern, Switzerland

e-mail: martin.kompis@insel.ch

\section{Introduction}

In clinical practice, patients with non-organic hearing losses pose a challenge in more ways than one. Before an adequate and ethical procedure for a patient with nonorganic hearing loss can be envisaged, it must be diagnosed with an acceptable reliability and sensitivity. Substantial differences in the frequency of the diagnosis of nonorganic hearing loss among different centers [1] suggest that this diagnosis may be missed in a non-negligible number of cases.

There are at least two reasons why it is important to identify non-organic hearing losses as early as possible. First, an unnecessary prolongation of suffering may result, if for e.g. a psychogenic hearing loss in a child is not recognized and the underlying cause, such as a serious problem at school, is not solved. Second, undetected nonorganic hearing losses can cause considerable expenses for useless or even harmful hearing aid fittings or if gainful employment is claimed to be impossible.

Especially in adults and older children, the results of psychoacoustical measurements are often not verified by objective measurements. It is therefore important to be aware of the typical features of a non-organic hearing loss to be able to administer specific tests if such a suspicion arises.

It is difficult to study the features of non-organic hearing losses in controlled experimental studies and a major part of our current knowledge on this topic is based on case descriptions. Amazingly, although non-organic hearing losses do not seem to be exceedingly rare, the cumulative number of cases reported in literature reaches only about 300 [1-11]. This figure includes both adults and children. As most of these studies encompass only a limited number of subjects, some features may be overlooked or their frequency of occurrence may be over- or underestimated. 
The aim of the present work was to broaden the knowledge of non-organic hearing loss by describing the audiometric findings to help diagnose non-organic hearing losses early in children and in adults and by increasing the cumulative number of patients described in scientific literature.

\section{Nomenclature}

The general terms "non-organic hearing loss" or "pseudohypacusis" describe a hearing loss, which can be found in an audiometric evaluation but cannot be explained by an organic disorder [12], or in which substantially poorer hearing thresholds are found in the subjective than in the objective measurements [7].

Usually, non-organic hearing losses are grouped into three distinct categories: malingering, aggravation and psychogenic hearing loss [13-15]. In the malingering category, a hearing loss is consciously feigned by a person with bilaterally normal hearing. In the aggravation category, an organic hearing loss is present, but the patient consciously wants the examiner and other persons to believe that the hearing loss is greater than it is in reality. In a psychogenic or functional hearing loss, the patient believes in having the hearing loss that she or he reports, though there is no organic hearing loss present. This last category of non-organic hearing losses is mostly attributed to children. Although this classification may have its shortcomings, in the absence of a better system we adhere to this prevailing classification [13-15] in this study.

\section{Materials and methods}

Patient records of the Division of Audiology of the University ENT Department of Bern, Switzerland, were reviewed over a 6 year period (2003-2008). In this period, 19,353 patient contacts took place. From these, a nonorganic hearing loss was diagnosed in 40 cases, where a diagnosis includes confirmation with at least one objective measurement (otoacoustic emissions or auditory-evoked potentials) and at least one pure tone audiogram showing substantially poorer hearing thresholds. In all cases, in which only otoacoustic emissions and no auditory-evoked potentials had been measured, auditory neuropathy was excluded on the basis of later measurements with normal pure tone and speech audiometric results.

The following data of the patient records were analyzed: age at the time of presentation, sex, findings in pure tone audiometry, findings in speech audiometry, results of objective measurements, use or prescription of hearing aids before the diagnosis of a non-organic hearing loss and accompanying circumstances such as reported problems at school.

Audiometric measurements were performed in a soundproof chamber (type 402A, Industrial Acoustics Company, Niederkrüchten, Germany) for all adults and in a special, soundproof and friendly room adapted to audiometric testing in children. Clinical audiometers (GSI 61, Grason-Stadler, Milford, NH, USA) were used for all measurements and calibrated according to the requirements set by the Swiss Commission for Audiology [16]. For speech audiometry, German two-digit numbers and monosyllabic words from the Swiss recording of the Freiburg test were used [16]. Speech audiograms were analyzed with regard to the following two criteria: (1) internal consistency, and (2) consistency with the pure tone audiogram of the same ear. The main aspect of internal consistency (1) is that monosyllabic words are never easier to understand than two-digit numbers, regardless of the presentation level. A speech audiogram was considered inconsistent with the pure tone audiogram (2) if the comparison between the increase of the presentation level for $50 \%$ understanding for two-digit numbers compared to normal hearing subjects and the pure tone average threshold at 250,500 , and $1,000 \mathrm{~Hz}$ differed by $15 \mathrm{~dB}$ or more. Brainstem auditory-evoked potentials were recorded either with a Navigator Pro or the older EP 4.00 system (both by Bio-logic Systems Corp., Mundelein IL, USA) using repetitive clicks of $100 \mu$ s duration without addition of notched noise.

If a unilateral hearing loss was presented, the Langenbeck test [17] and the Stenger test [18] were routinely administered. Instead of the tuning forks suggested in the original publication, an audiometer was used [15].

\section{Results}

Distribution according to age and sex

The subjects included 22 children and adolescents (ages 716 , average 10.6 years) and 18 adults (ages 19-57, average 39.7 years). Figure 1 shows the frequency of occurrence versus age. Table 1 shows a synopsis of the sex and diagnoses. As much as $64 \%$ of the children and $61 \%$ of the adults were female. In children, there is a peak at age 11 years, and $86 \%$ of the children are between ages 8 and 13 at the time of diagnosis. There seems to be a more even distribution in adults in the range of 19-57 (average: 39.7) years. In our adult group, there was no person over the age of 57 , i.e. after retirement or in the last few years before retirement. 


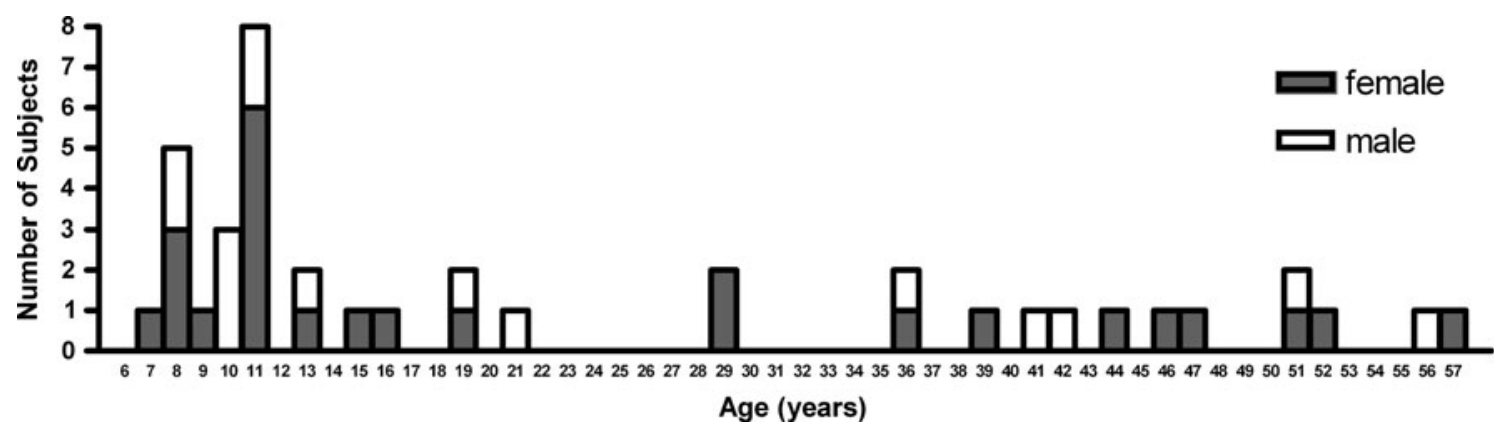

Fig. 1 Distribution of non-organic hearing loss with age and sex

Table 1 Distribution of the different categories of non-organic hearing loss with age group and sex

\begin{tabular}{llllll}
\hline Diagnosis & \multicolumn{2}{l}{ Children } & & & Adults \\
\cline { 2 - 3 } \cline { 6 - 6 } & Female & Male & & Female & Male \\
\hline Aggravation & 0 & 0 & & 5 & 4 \\
Malingering & 0 & 0 & & 2 & 3 \\
Psychogenic & 14 & 8 & & 4 & 0 \\
\hline
\end{tabular}

Children

All 14 girls and 8 boys included in this study were diagnosed with a psychogenic hearing loss. In 20 children, pure tone audiogram suggested a bilateral hearing loss; in two the hearing loss was unilateral. In all children, a bilaterally normal hearing was found using objective measurements. All 22 children had normal click-evoked otoacoustic emissions in both ears. In 12 children, auditory-evoked potentials were measured and found to be bilaterally normal. Normal pure tone audiograms were measured at a later stage in 14 of the 22 children. In eight cases, their parents either refused or did not show up for a follow-up examination.

\section{Pure tone audiograms}

Figure 2 shows a synopsis of pure tone audiograms of the 22 children. Most of the audiograms were flat, some of them remarkably so. Nevertheless, occasionally a usually mild drop or increase toward higher frequencies was seen. Most thresholds were found between 30 and $80 \mathrm{~dB}$ with a possible maximum around $50 \mathrm{~dB}$. If the hearing loss was bilateral, the difference between the thresholds of the two ears was $15 \mathrm{~dB}$ or less in $75 \%$ of the children. Most audiograms suggested a small air-bone gap of 5-25 dB, as shown in Fig. 4 and discussed in detail later.

In 11 of the 22 children, more than one audiogram showing a non-organic hearing loss was available. In all cases, reproducibility of the hearing thresholds between the audiograms was poorer than expected in organic hearing loss.

\section{Speech audiometry}

In 12 of the 22 children, speech audiometry was performed and documented. In 11 children, the speech audiogram suggested a considerably better pure tone threshold than the pure tone audiogram. In one child, the speech audiogram was internally inconsistent according to the criteria given above.

\section{Concomitant circumstances}

A number of concomitant circumstances, which are believed to be particularly frequent in children with nonorganic hearing loss, have been identified by several authors [2, 8, 19]. Table 2 shows a synopsis of the circumstances found in our study group.

Four children had previous experience with a transient hearing loss, nine knew a hard-of-hearing person, often one of the grandparents, and in two children a coincidence of both of these concomitant circumstances was found. In 18 cases, a conflict or a difficult situation at home, school or both were identified. In school, difficult relationships with teachers and problems with classmates were reported. At home, a larger variety of difficult circumstances, including divorce of the parents, other changes in the configuration of the family or a serious illness of a sibling occurred. In four cases, no conflict was found. Note that even if a conflict can be identified, which appears to be the reason for the non-organic hearing loss, there are no scientifically verifiable criteria to prove a causal connection.

Normal conversation without the use of hearing aid was observed and documented in 21 children. In most cases, this was in obvious contrast to the substantial deafness found in the audiogram. Three children had been fitted with hearing aids before the first presentation at our center, but none of them used the devices on a regular basis. One child showed a marked interest in medical examinations. She had also requested an ophthalmologic examination earlier, claiming that she was not seeing well. The examination did not reveal any abnormalities of the visual system. 
Fig. 2 Hearing thresholds in children with non-organic hearing loss: a air conduction threshold (average of both ears for bilateral hearing loss, threshold of poorer ear for unilateral loss), b difference between the side with better and that with poorer threshold for bilateral hearing loss (a) Hearing threshold

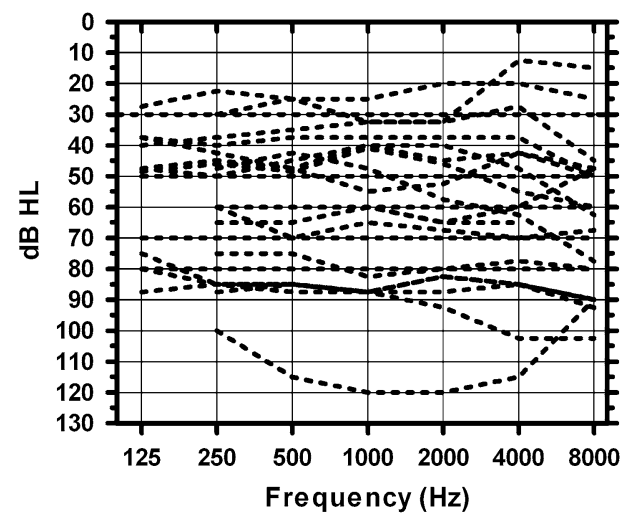

(b) Difference between ears

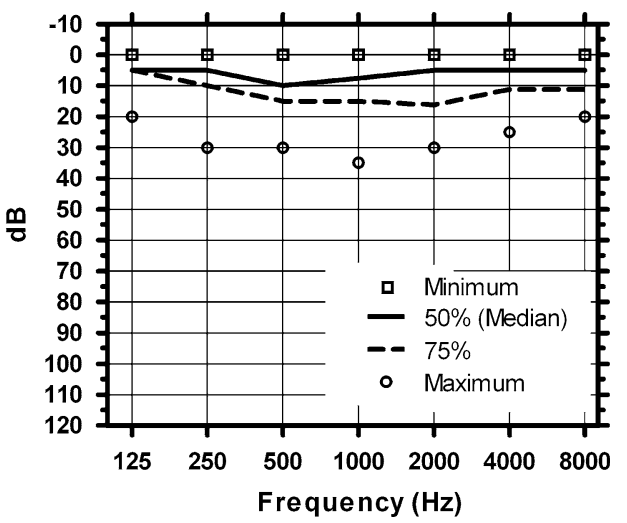

Table 2 Frequency of occurrence of several concomitant circumstances in 22 children with a non-organic hearing loss

\begin{tabular}{lc}
\hline Concomitant circumstances & Number of occurrences \\
\hline History of middle ear problem with transient hearing loss & $6(27 \%)$ \\
Child knows a hard-of-hearing person & $11(50 \%)$ \\
Conflict or difficult situation both at home and in school & $4(18 \%)$ \\
Conflict or difficult situation at home, but not in school & $4(18 \%)$ \\
Conflict or difficult situation in school, but not at home & $10(45 \%)$ \\
Skipped one grade in school & $1(5 \%)$ \\
Normal conversations possible without hearing aids & $21(95 \%)$ \\
Child asked for other ophthalmologic examination & $1(5 \%)$ \\
Has been fitted with hearing aids & $3(14 \%)$
\end{tabular}

Adults

Besides the psychoacoustic tests described below, the diagnosis of a non-organic hearing loss in adults was based on the measurement of otoacoustic emissions and auditoryevoked potential in 11 patients, on otoacoustic emission in 5 patients and an auditory-evoked potentials in 2 patients.

In contrast to the group of children, only 4 (22\%) of the non-organic hearing losses were diagnosed as psychogenic among the adults (Table 1). Nine cases (50\%) were attributed to aggravation of an existing, but less pronounced, organic hearing loss and five were diagnosed as malingering. A purely unilateral non-organic hearing loss was found in five adults (three malingering, one psychogenic, one aggravation).

To differentiate between malingering and psychogenic hearing loss, in principle, the treating physician must know whether the patient really believes him- or herself to be hard of hearing or not. However, unless the patient openly admits at some stage to having consciously simulated his or her hearing loss (3 patients of our group), there is no infallible method to achieve this. In the absence of agreed criteria, in our group, psychogenic hearing loss was diagnosed when: the patient did not obviously try to hide his normal hearing capability in the conversations before and after the audiologic examination (2 patients); the treating psychiatrist stated that the psychogenic hearing loss was in line with the underlying psychiatric problem ( 2 patients). In the absence of an open confession, malingering was diagnosed when a normal audiogram was obtained within the same session after an explanation of the first conflicting results of the examination and its potential legal implications (1 patient) and in one patient applying for disability benefits, when she aborted the consultation mid-sentence, after the obvious discrepancy in the data collected previously was explained to her.

\section{Pure tone audiograms}

Figure 3 shows a synopsis of the pure tone audiograms found in 18 adults. As shown in Fig. 3a, thresholds are mostly in the range of $40-60 \mathrm{~dB}$ and tend to be relatively constant with frequency. However, exceptions did occur showing both increasing and decreasing thresholds above 1,000-2,000 Hz. Air-bone gaps usually lay between 0 and $15 \mathrm{~dB}$ and were never found to be negative. In 12 of the 18 patients, an unusually poor reproducibility of the hearing thresholds during the test was documented. 
Fig. 3 Hearing thresholds in adults with non-organic hearing loss: a air conduction threshold (average of both ears for bilateral hearing loss, threshold of poorer ear for unilateral loss; dotted lines: psychogenic, dashed lines: malingering, solid lines: aggravation). b Difference between the side with better and that with poorer threshold for bilateral hearing loss (a) Hearing threshold

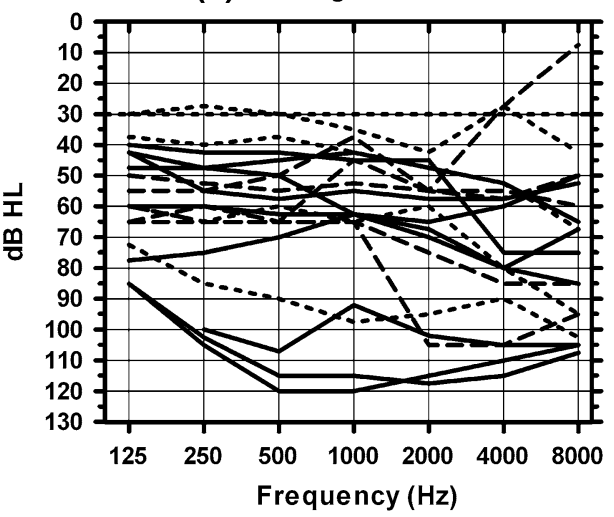

(b) Difference between ears

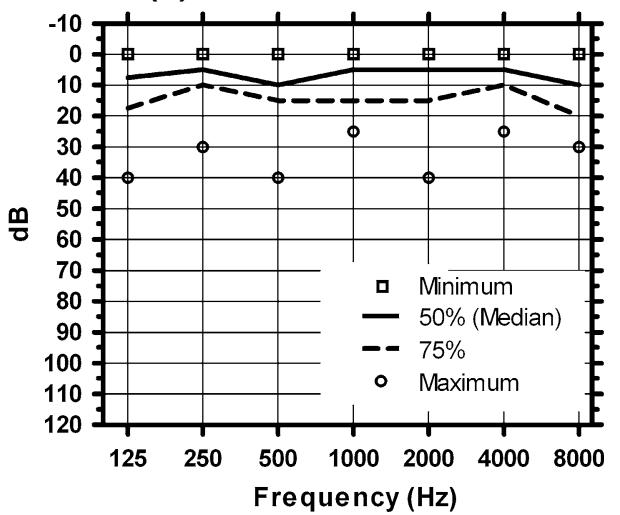

Speech audiograms

Speech audiometry was performed in 13 of the 18 adults. Table 3 shows a synopsis of the findings. In three subjects, the speech audiogram was rated as internally inconsistent and therefore implausible. In another six subjects, the speech reception threshold for two-digit numbers was either too good (4 subjects) or too poor ( 2 subjects) to be consistent with the pure tone audiometric threshold, the difference between the two being $15 \mathrm{~dB}$ or more. In four subjects, speech audiograms were internally consistent and reasonably consistent (i.e. within $\pm 15 \mathrm{~dB}$ ) with the pure tone audiogram. In all subjects presenting with a binaural hearing loss, speech audiograms on both sides were in the same category as listed in Table 3. Although the numbers in our subgroups are too small for a conclusive statistical analysis, there is an intriguing cumulation of inconspicuous speech audiograms in the psychogenic hearing loss group only.

\section{Langenbeck and Stenger test}

In all five patients presenting with a unilateral hearing loss, a Stenger and a Langenbeck test $[15,17,18]$ were administered. In all five patients, the result of both tests indicated a non-organic hearing loss.
Use of hearing aids

Seven adults were fitted with a hearing aid. One subject was diagnosed as malingering, and had stopped using his hearing aid at the time of the first presentation at our center. Two subjects were in the psychogenic hearing loss group and four were diagnosed with aggravation. In three of the last four patients, a hearing aid would have been probably prescribed on the basis of the organic hearing loss alone.

\section{Psychiatric treatment at the time of presentation}

Three of the adult subjects, two of them diagnosed with a psychogenic hearing loss and one with aggravation, were undergoing psychiatric treatment at the time of their first presentation at our center. The reasons for their treatment were depression in two cases and a somatization disorder in one case.

\section{Suspected reasons for non-organic hearing loss}

A probable cause for non-organic hearing loss was found in 10 of the 18 adults. In two patients with a psychogenic hearing loss, the underlying problem was believed to be associated with the psychiatric condition being actively treated at that time. Three subjects went through difficult

Table 3 Synopsis of speech audiometric findings in adults

\begin{tabular}{|c|c|c|c|c|}
\hline Finding & Number of subjects & Thereof malingering & Thereof aggravation & Thereof psychogenic \\
\hline Internally inconsistent speech audiogram & $3(17 \%)$ & 1 & 2 & 0 \\
\hline Speech audiogram, good ${ }^{\mathrm{a}}$ & $4(22 \%)$ & 1 & 3 & 0 \\
\hline Speech audiogram, poor $^{\mathrm{a}}$ & $2(11 \%)$ & 1 & 1 & 0 \\
\hline Internally consistent and plausible speech audiogram & $4(22 \%)$ & 0 & 1 & 3 \\
\hline (No speech audiogram available) & $5(28 \%)$ & 2 & 2 & 1 \\
\hline Total & $18(100 \%)$ & 5 & 9 & 4 \\
\hline
\end{tabular}

${ }^{\text {a }}$ Difference of $15 \mathrm{~dB}$ or more relative to the threshold in pure tone audiogram 
phases in their lives (one recent diagnosis of cancer, one unplanned and unexpected parenthood, one difficult job situation).

In five subjects, financial reasons seemed to be the cause of non-organic hearing loss. One subject had general financial problems, three had applied for disability benefits and one subject hoped to get a higher financial contribution toward her hearing aid from her insurance by presenting an aggravation of her hearing problem.

\section{Discussion}

Pure tone thresholds

Pure tone audiograms in children and adults are usually flat and thresholds are frequently between 40 and $70 \mathrm{~dB}$. However, lower as well as considerably higher thresholds do occur in both groups. Our study confirms these previous findings [13-15].

\section{Apparent air-bone gap}

In both groups, a small air-bone gap (ABG) of usually 5$20 \mathrm{~dB}$ can be found frequently. The $\mathrm{ABG}$ tends to be larger at lower frequencies. Figure 4 shows a synopsis of the data of all 40 subjects.

We believe that this is a specific feature of non-organic hearing loss, which, to our knowledge, has not been described before. We propose the following explanation, based on the different loudness growth, for air conduction (AC) and bone conduction (BC). As the test tone is well above the real, organic hearing threshold, the beginning activation of the stapedial reflex may provide a small damping for AC measurements only. For BC measurement, the stapedial reflex will be activated similarly; but as the sound energy is not transmitted through the middle ear, test

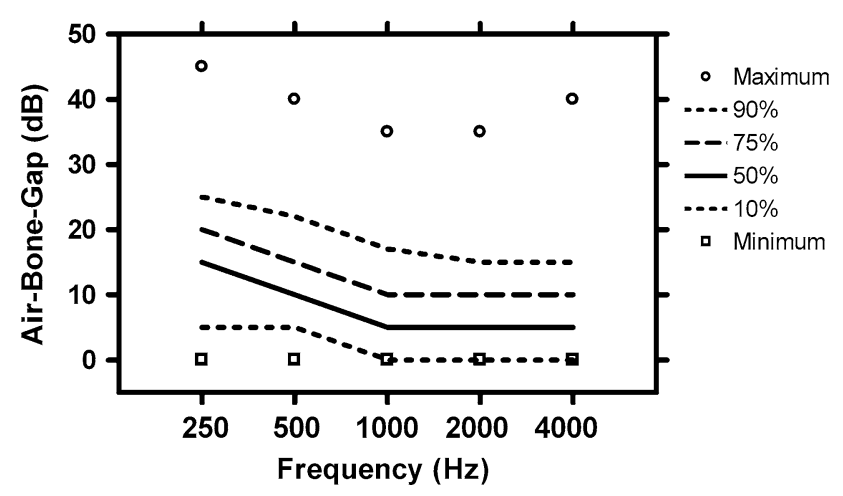

Fig. 4 Synopsis of the apparent air-bone gap found in the audiograms of 40 subjects with non-organic hearing loss tones via $\mathrm{BC}$ will be perceived slightly louder at the same dial reading of the audiometer, when compared to those presented by AC. We expect the effect to be somewhat larger at lower frequencies and greater than the damping alone, as there may be an additional increase in subjective loudness due to an internal occlusion effect [20] caused by the stapedial reflex.

This finding may add to the diagnostic value of stapedial reflex measurements in non-organic hearing losses. Stapedial reflexes are expected to appear at higher stimulation thresholds or even to become immeasurable altogether if there is a substantial, organic air-bone gap present, but not in non-organic hearing losses.

Non-organic hearing loss in children

Non-organic hearing losses in children seem to occur mostly in the age range of approximately 8-13 years and are found more often in girls than in boys. This is in agreement with earlier studies [9, 19]. In an earlier report, Graf [2] summarized several signs that can be found in children with psychogenic hearing loss:

1. the child knows a person with a hearing problem or has a history of a transient hearing disorder;

2. the parents or a teacher suspects a hearing loss;

3. conflict at school or at home;

4. intelligence is normal or better;

5. symmetric, flat hearing loss of $40-80 \mathrm{~dB}$;

6. normal conversation possible outside of the test situation.

Our data (Fig. 2; Table 2) supports the notion that these points are still valid after more than 40 years. In our study group, all but two children showed bilateral hearing loss. While several earlier studies also report predominantly or even exclusively bilaterally increased hearing thresholds in children [8, 11], at least in one study non-organic hearing losses in children were found to be predominantly unilateral (12 of 20 children [10]). We did not find a convincing explanation for this difference among the different studies.

Non-organic hearing loss in adults

In adults, non-organic hearing loss was found at all ages between 19 and 57 years, possibly with a very slight peak in the early 40s. It is interesting to note that so far we have seen no subjects with a non-organic hearing loss in the retirement age and are not aware of any such report in literature. We hypothesize that a number of potential reasons for a non-organic hearing loss disappear together with the need of gainful employment. Non-organic hearing losses in adults seem to be associated with the active middle age. 
Of the 18 adults included in our study, 5 (28\%) presented a unilateral hearing loss. This is somewhat more than reported in an earlier study by Balatsouras et al. (12.5\%) [7]. The difference is probably due to the relatively small numbers of subjects in both studies.

\section{Speech audiometry}

Speech audiometry has been found to be a valuable tool when confronted with a non-organic hearing loss. It is well known that speech audiometry tends to show better results than those expected from pure tone thresholds [1, 3, 7, 12]. Contrary to this conventional wisdom, two subjects in our study group showed speech reception thresholds, which were considerably poorer than expected on the basis of pure tone audiometry. To our knowledge, this is the first report describing this finding in non-organic hearing loss.

Four speech audiograms were reasonably consistent with the pure tone audiogram reflecting a non-organic hearing loss. A consistent speech audiometric finding alone is therefore clearly insufficient to eliminate the diagnosis of a non-organic hearing loss.

\section{Conclusions}

With the wide availability of objective measurements such as otoacoustic emissions and auditory evoked potentials, today the basis for diagnosing nonorganic hearing losses is a discrepancy between poor psycho-acoustically measured hearing threshold and better threshold from the objective measurements. Nevertheless, there are a number of typical signs, which can be found in routine clinical examinations. These signs include almost frequency-independent audiograms showing sensorineural hearing losses, often with a small air-bone gap, inconsistent speech audiograms and, in children, previous knowledge about hearing losses. Speech audiograms may be internally inconsistent or suggest a considerably better or considerably poorer hearing than documented by pure tone audiogram. As no single sign is compulsory, it is useful to be aware of the multitude of typical signs to be able to actively confirm or eliminate a suspected non-organic hearing loss.

Acknowledgments We would like to thank Birgit Bormann and Martin Krebs for their valuable contributions and Ms Eva Clamann for her help in preparing this text.

Conflict of interest statement The authors have no conflict of interest to declare.

\section{References}

1. Zorowka PG (1992) Psychogene Hörstörung im Kindes- und Jugendalter. HNO 40:386-391

2. Graf K (1966) Die nichtorganische Schwerhörigkeit beim Kind. Pract oto-rhino-laryng 28:20-30

3. Feldmann H (1986) Das Bild der psychogenen Hörstörung heute. Laryngo-Rhino-Otol 68:249-258

4. Jahnke K, Solzbacher D (2005) Simulation von Taubheit im Gutachtenwesen. Laryngo-Rhino-Otol 84:511-515

5. Yamamoto M, Kanzaki J, Ogawa K, Asano K (1991) Psychological aspects of psychogenic deafness in children. Int J Pediatr Otorhinolaryngol 21:113-120

6. Qui WW, Stucker FJ, Yin SS, Welsh LW (1998) Current evaluation of pseudohypacusis: strategies and classification. Ann Otol Rhinol Laryngol 107:638-647

7. Balatsouras DG, Kaberos A, Korres S, Kandiloros D, Ferekidis E, Ecounomou C (2003) Detection of pseudohypacusis: a prospective, randomized study of the use of otoacoustic emissions. Ear Hear 24:518-527

8. Pracy JP, Walsh RM, Mepham N, Bowdler DA (1996) Childhood pseudohypacusis. Int J of Pediatric Otorhinolaryngol 37:143-149

9. Saravanappa N, Mepham GA, Bowdler DA (2005) Diagnostic tools in pseudohypacusis in children. Int $\mathrm{J}$ Ped Otorhinolaryngol 69:1235-1238

10. Hosoi H, Tsuta Y, Murata K, Levitt H (1999) Suggestion audiometry for non-organic hearing loss (pseudohypacusis) in children. Int J Pediatr Otorhinolaryngol 47:11-21

11. Kothe C, Fleischer S, Breitfuss A, Hess M (2003) Diagnostik von psychogenen Hörstörungen im Kindesalter. HNO 51:915-920

12. Lin J, Staecker H (2006) Nonorganic hearing loss. Semin Neurol 26:321-330

13. Böhme G, Welzl-Müller K (2005) Simulation, aggravation, dissimulation, psychogene hörstörungen. In: Lehrbuch Audiometrie, Hörprüfungen im Erwachsenen- und Kindesalter. 5th edn. Hans Huber, Göttingen, pp 112-115

14. Lehnhardt E (2001) Aggravation, Simulation, psychogene Hörstörung. In: Praxis der Audiometrie. 8th edn. Thieme Stuttgart pp 239-250

15. Kompis M (2009) Psychogene Hörstörungen, Simulation, und Aggravation. In: Audiologie. 2nd edn. Hans Huber Göttingen pp 183-190

16. Kompis M, Krebs M, Häusler R (2006) Überprüfung der Bezugskurven der Schweizer Version des Freiburger Zahlen- und Einsilbertests. HNO 54:445-450

17. Langenbeck B (1933) Was beweist der Stengersche Versuch? Z Hals Nas u Ohr Heilk 34:250

18. Stenger S (1900) Ein Versuch zur objektiven Feststellung einseitiger Taubheit bzw. Schwerhörigkeit mittels Stimmgabeln. Arch Ohrenheilk 50:197-198

19. Radkowski D, Cleveland S, Friedmann EM (1998) Childhood pseudohypacusis in patients with high risk for actual hearing loss. Laryngoscope 108:1534-1538

20. Reinfeld S, Stenfelt A, Good T, Hakansson B (2007) Examination of bone-conducted transmission from sound field excitation measured by thresholds, ear-canal sound pressure, and skull vibration. J Acoust Soc Am 121:1576-1587 